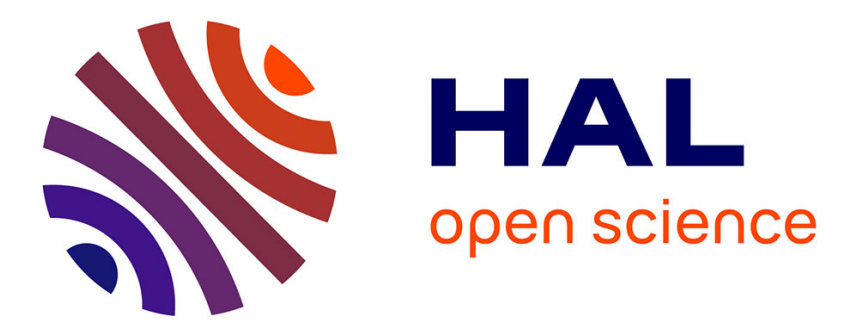

\title{
Effect of side chain modifications in imidazolium ionic liquids on the properties of the electrical double layer at a molybdenum disulfide electrode
}

Zheng Gong, Agílio Pádua

\section{- To cite this version: \\ Zheng Gong, Agílio Pádua. Effect of side chain modifications in imidazolium ionic liquids on the properties of the electrical double layer at a molybdenum disulfide electrode. Journal of Chemical Physics, 2021, 154, pp.084504. 10.1063/5.0040172 . hal-03135629}

\author{
HAL Id: hal-03135629 \\ https://hal.science/hal-03135629
}

Submitted on 23 Feb 2021

HAL is a multi-disciplinary open access archive for the deposit and dissemination of scientific research documents, whether they are published or not. The documents may come from teaching and research institutions in France or abroad, or from public or private research centers.
L'archive ouverte pluridisciplinaire HAL, est destinée au dépôt et à la diffusion de documents scientifiques de niveau recherche, publiés ou non, émanant des établissements d'enseignement et de recherche français ou étrangers, des laboratoires publics ou privés. 


\title{
Effect of side chain modifications in imidazolium ionic liquids on the properties of the electrical double layer at a molybdenum disulfide electrode
}

\author{
Zheng Gong and Agilio A. H. Padua a) \\ Laboratoire de Chimie, École Normale Supérieure de Lyon $\&$ CNRS, 69364 Lyon, \\ France
}

(Dated: 28 January 2021)

\begin{abstract}
Knowledge of how the molecular structures of ionic liquids (ILs) affect their properties at electrified interfaces is key towards the rational design of ILs for electric applications. Polarizable molecular dynamics simulations were performed to investigate the structural, electrical and dynamic properties of electric double layers (EDLs) formed by imidazolium dicyanamide ([ImX1][DCA]) at the interface with molybdenum disulfide electrode. The effect of side chain of imidazolium on the properties of EDLs was analyzed by using 1-ethyl-3-methylimidazolium ([Im21]), 1-octyl-3-methylimidazolium ([Im81]), 1-benzyl-3-methylimidazolium ([ImB1]) and 1-(2-hydroxyethyl)-3-methylimidazolium ([ImO1]) as cations. Using [Im21] as reference, we find that the introduction of octyl or benzyl groups significantly alters the interfacial structures near the cathode because of the reorientation of cations. For [Im81], the positive charge on the cathode induces pronounced polar and non-polar domain separation. In contrast, the hydroxyl group has minor effect on the interfacial structures. [ImB1] is shown to deliver slightly larger capacitance than other ILs even though it has larger molecular volume than [Im21]. This is attributed to the limiting factor for capacitance being the strong association between counter-ions, instead of the free space available to ions at the interface. For [Im81], the charging mechanism is mainly the exchange between anions and octyl tails, while for the other ILs, the mechanism is mainly the exchange of counter-ions. Analysis on the charging process shows that the charging speed does not correlate strongly with macroscopic bulk dynamics like viscosity. Instead, it is dominated by local displacement and reorientation of ions.
\end{abstract}

\section{INTRODUCTION}

Two-dimensional (2D) nanomaterials such as graphene, hexagonal boron nitride and transition metal dichalcogenide are composed of atomically-thin layers assembled by means of weak, non-covalent forces. Due to electron confinement in two dimensions, 2D materials are able to access unprecedented physical, electronic and chemical properties, therefore be compelling in applications of novel electronic devices and sensors. ${ }^{1,2}$ In electrical applications such as batteries, electrical double layer capacitors (EDLCs) and fieldeffect transistors (FETs), novel electrolytes are required to function together with 2D nanomaterials. Roomtemperature ionic liquids (ILs) have unique properties, such as wide electrochemical window, high thermal stability, low vapor pressure, making them promising electrolytes. ${ }^{3-7}$

At the electrode-electrolyte interfaces, the ILs form ultra-thin electrical double layers (EDLs). The structure and dynamics of EDLs are among the key factors determining the capacitance and charging speed of EDLC and the gating speed of FET, and thus the performance of the devices. A fundamental understanding of the behavior of EDLs from the molecular level is essential for designing electrolytes with improved performance. In recent decades, numerous research efforts have been reported

a)Electronic mail: agilio.padua@ens-lyon.fr on EDLs formed at the IL-electrode interfaces, consisting in experimental, ${ }^{8}$ mean-field theory ${ }^{9}$ and simulation ${ }^{10-12}$ studies.

Molecular dynamics (MD) simulation is a powerful tool for investigating the properties of EDLs. It has provided major insights into the structure of the interfacial layers with atomic resolution, helped understand voltagecapacitance relations and the charging mechanisms. ${ }^{13-18}$ Extensive works have focused on how the properties of EDLs will be affected by the nature of the electrodes, e.g. the curvature, ${ }^{19,20}$ the roughness ${ }^{21,22}$ and the presence of nanopores. ${ }^{14,15}$ Notably, it has been shown that nanoporous carbon electrodes could deliver much larger capacitance than planar graphite electrode because the confinement of ions inside the pores suppresses the overscreening effect and allows ions to approach the electrode surface more closely. ${ }^{14}$

The molecular structures of electrolytes is of comparable importance to that of electrodes in determining the properties of EDLs, e.g. imidazolium- and pyridiniumbased ILs are shown to provide larger capacitance but lesser electrochemical stability than ammonium-based ILs. ${ }^{23}$ The functionalization of ions offers very broad possibilities to tune the properties of ILs at interfaces. For a planar electrode, it is usually believed that ILs with smaller ion size are likely to deliver larger interfacial capacitance, because of the more compact packing near the electrodes and thinner EDLs, as shown both by experiments ${ }^{23,24}$ and simulations. ${ }^{25}$ However, it was recently reported that bulky surface-active an- 
ions, such as 1,4-bis(2-ethylhexoxy)-1,4-dioxobutane-2sulfonate and dodecyl sulfate, actually deliver larger capacitances than tetrafluoroborate at high temperature, ${ }^{26}$ and this result was obtained using the same 1-butyl-3methylimidazolium cation. This enhancement was attributed to self-assembly of the surface active anions into lamellar structures that suppress the over-screening effect. This finding emphasizes that the microscopic structures of ions can have a prominent effect on the properties of EDLs, and thus may affect the performance of electric devices dramatically.

In order to rationally design ILs for specific electric applications, it is therefore of particular interest to understand how modifications in the side chains will affect the properties of EDLs. So far, most of the reports focused on the effect of different lengths of alkyl tails, ${ }^{24,25,27-31}$ suggesting that longer side chain results in smaller capacitance and triggers the change of the voltage-capacitance curve from bell-shape to camel-shape. Other than the alkyl tail, the effect of introducing ether groups (increasing the polarity of the side chains) has been considered, showing that the tail containing ether groups is more likely to be parallel to the electrode surface in the first layer than alkyl tails, thus indicating stronger adsorption between ether-functionalized tail on the electrode. ${ }^{32}$

In this work, we investigate the effects of different sizes and chemical functions in side chains upon the local ordering and properties of EDLs formed by alkylmethylimidazolium dicyanamide $([\operatorname{ImX} 1][\mathrm{DCA}])$ electrolytes at planar $\mathrm{MoS}_{2}$ electrodes using polarizable MD simulations. Four cations are considered: 1-ethyl-3-methylimidazolium ([Im21]), 1-octyl-3-methylimidazolium ([Im81]), 1-benzyl-3methylimidazolium ([ImB1]) and 1-(2-hydroxyethyl)-3methylimidazolium ([ImO1]), as depicted in Fig. 1. The ethyl, octyl, benzyl and 2-hydroxyethyl groups are representative for different shape, size and hydrophobicity.

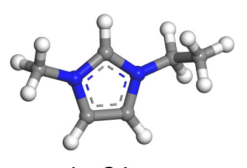

$\operatorname{lm} 21$

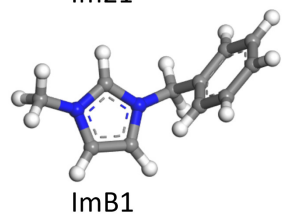

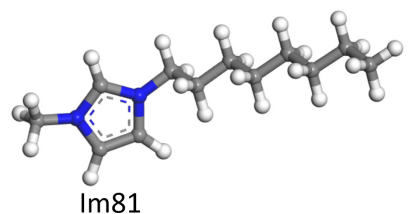
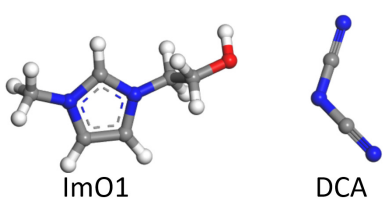

FIG. 1. Structures of ions considered in this work.

$\mathrm{MoS}_{2}$ is used as electrode here because of its promising application in FETs based on 2D nanomaterials. ${ }^{33-36}$ In IL-gated FETs, the electrostatic field exerted by the EDL induces doping on the semiconducting flake $\mathrm{MoS}_{2}$ electrodes by stabilizing charge carriers with a density up to $10^{14} \mathrm{~cm}^{-2} \cdot{ }^{37} \mathrm{~A}$ high capacitance at the electrolytic interface is linked to a high carrier density in the semicon- ductor, albeit with a lower carrier mobility. ${ }^{38,39}$ These quantities are strongly dependent on the nature of the ILs, which is an argument towards optimizing the electrolyte through judicious choice of the chemical structure of the ions. Recently, the gating effect of amorphous indium-gallium-zinc oxide FET coupled with a series of imidazolium-based ILs was investigated, demonstrating that both charge density and homogeneity of charge distribution at the electrode surface are important to the gating performance. ${ }^{40}$ In another work, the solvent effect on $\mathrm{MoS}_{2}$ FET gated by a mixture of 1-butyl3 -methylimidazolium tetrafluoroborate and acetonitrile was studied, showing that the concentration of acetonitrile has a non-monotonic influence on the gating speed. ${ }^{41}$

The polarization effect is pronounced in ILs because of the strong local electric field. Although electrode polarization has been taken into account in recent simulation works on EDLs, the electrolytes are usually described by non-polarizable or even coarse-grained models, with only a few exceptions. ${ }^{42,43}$ Recent advances in GPUaccelerated MD simulation make it possible to describe the electrified interface with more detailed models. ${ }^{44,45}$ In this work, full polarization is considered for both electrode and electrolytes by utilizing a constant-voltage simulation method together with the recently developed CL\&Pol polarizable force field for ionic liquids. ${ }^{46}$

\section{MODELS AND METHODOLOGIES}

Constant-voltage simulations were performed to resemble the experimental conditions. That is, a voltage drop is pre-assigned between electrodes, while charge accumulated on the electrode is an observable, in contrast with the simulation condition where a constant charge density is pre-assigned to the electrodes and held fixed during the simulation. We note that since $\mathrm{MoS}_{2}$ is a semiconductor, the constant charge density setup is likely to be more realistic than constant voltage method, which is strictly valid only for ideal conductor. However, recent works have shown that these two setups give similar interfacial structures and capacitances under low voltage drops ${ }^{42,43,47}$ while a unique advantage of the constant voltage method is that it allows us to simulate the charging process more accurately. ${ }^{48}$ At the beginning of charging, a voltage drop is suddenly applied and charge starts accumulating at the electrode. The difficulty for constant-charge simulation during the charging process is that the correct charge density to be assigned on the electrode at each timestep is unknown.

The three-dimensional periodic image charge method recently proposed by Dwelle et al..$^{49}$ was used in this work for constant-voltage simulations. Fig. 2 illustrates the geometry of the simulation box with ionic liquid confined between two $\mathrm{MoS}_{2}$ plane electrodes. The electrodes are oriented along the $x y$ plane and separated by $d=8.0 \mathrm{~nm}$, which we measure as the distance between the $\mathrm{S}$ atoms in contact with ionic liquids on the cathode and the anode. 
Considering the two electrodes as mirrors, each atomic charge in the electrolyte creates two oppositely signed image charges with respect to the two mirror planes. The image charges then generate higher-order image charges with respect to the mirrors, which can be captured by utilizing three-dimensional periodic boundary conditions with a length in the $z$ direction of $2 d=16.0 \mathrm{~nm}$. By applying an external electric field $E_{\text {ext }}$ on the electrolyte atoms, the voltage drop $\Delta V$ between two electrodes is guaranteed. ${ }^{49}$

$$
E_{\text {ext }}=\frac{\Delta V}{d}
$$

Each electrode contains two layers of $\mathrm{MoS}_{2}$ made of $13 \times 8$ unit cells. The length of simulation box in $x$ and $y$ directions are 4.109 and $4.380 \mathrm{~nm}$, respectively. During image charge simulation, the electrode atoms are restrained at their original positions by a harmonic potential so that the locations of mirrors does not change.

$U_{\mathrm{res}}=\sum_{i}^{\mathrm{MoS}_{2}}\left(k_{x}\left(x_{i}-x_{i, 0}\right)^{2}+k_{y}\left(y_{i}-y_{i, 0}\right)^{2}+k_{z}\left(z_{i}-z_{i, 0}\right)^{2}\right)$

herein, $x_{i}, y_{i}$ and $z_{i}$ are the position of each Mo and $\mathrm{S}$ atom. $x_{i, 0}, y_{i, 0}$ and $z_{i, 0}$ are the original position. $k_{x}$, $k_{y}$ and $k_{z}$ are set to $0.1,0.1$ and $500.0 \mathrm{kcal} \mathrm{mol}^{-1} \mathrm{~nm}^{-2}$, respectively. At each MD timestep, the positions of im- age charges are updated according to the coordinates of electrolyte atoms, immediately after the integration for positions.

The numbers of ion pairs for the systems studied are listed in Tab. I, which are determined based on the calculated densities of bulk ILs at $333 \mathrm{~K}$ and 1 bar. The simulation lengths are also listed in the table. Note that a trajectory of hundreds of nanoseconds is required to attain converged interfacial structures for these systems.

TABLE I. The number of ion pairs and trajectory length for each electrolyte.

\begin{tabular}{ccc}
\hline Electrolyte & Number & Length (ns) \\
\hline [Im21][DCA] & 511 & 180 \\
[Im81][DCA] & 312 & 480 \\
[ImB1][DCA] & 392 & 360 \\
[ImO1][DCA] & 503 & 240 \\
\hline
\end{tabular}

The ILs are modeled with the recently developed Drude polarizable force field CL\&Pol ${ }^{46,50}$ with several refinements, whereas the electrode atoms are modeled as charge neutral particles with only LJ interactions. The details of force field and simulation conditions are given in section V. Near the electrodes, there are strong attractions between Drude particles and their images, which cause instability if a Drude particle comes too close to the electrode. Therefore, a wall potential is applied to all Drude particles of ILs to avoid this situation:

$$
U_{\text {wall }}=\left\{\begin{array}{l}
\sum_{i}^{\text {Drude }} 4 \epsilon\left(\left(\frac{\sigma}{z_{i}-z_{+}}\right)^{12}-\left(\frac{\sigma}{z_{i}-z_{+}}\right)^{6}+0.25\right), z_{i}-z_{+}<1.1225 \sigma \\
\sum_{i}^{\text {Drude }} 4 \epsilon\left(\left(\frac{\sigma}{z_{-}-z_{i}}\right)^{12}-\left(\frac{\sigma}{z_{-}-z_{i}}\right)^{6}+0.25\right), z_{-}-z_{i}<1.1225 \sigma
\end{array}\right.
$$

herein, $\epsilon$ and $\sigma$ determine the strength and range of the wall potential, which are set to $0.5 \mathrm{kcal} \mathrm{mol}^{-1}$ and $0.15 \mathrm{~nm}$, respectively. The $z_{+}$and $z_{-}$coordinates are the locations of cathode and anode, which are at $0 \mathrm{~nm}$ and $8.0 \mathrm{~nm}$, respectively. We note that the wall potential is simply a safeguard. It decays to zero when the Drude particle is beyond $0.1684 \mathrm{~nm}$ from electrodes. The wall potential is only effective during the initial stage of the simulation when the interfacial strucure is evolving dramatically. By inspecting the converged trajectories, the minimum distance between Drude particles and electrode is $0.18 \mathrm{~nm}$ because of the $\mathrm{vdW}$ repulsion between heavy atoms. Therefore, this wall potential has no impact on the equilibrated trajectories.

\section{RESULTS AND DISCUSSION}

\section{A. Structure of the interfaces}

Fig. 3 shows the distributions of centers of mass (COM) of imidazolium heads of [Im21] cations and [DCA] anions along $z$ direction under voltage drops of 0 and $2 \mathrm{~V}$. In the absence of voltage drop, [Im21][DCA] forms layered structures at the interface which oscillate for around $1.5 \mathrm{~nm}$ before reaching the bulk liquid. The symmetry of this distribution profile confirms the convergence of the trajectories. Herein, we use the peaks of the distribution of [DCA] to identify the location of layers at the interface, because the imidazolium head group can adopt different orientations near the electrodes. Three layers can be identified, which are located at $0.34,0.72$ and $1.09 \mathrm{~nm}$ from the electrodes. The [Im21] are more likely than the $[\mathrm{DCA}]$ to be found in the first layer. As the voltage increases to $2 \mathrm{~V}$, the $[\operatorname{Im} 21]$ and $[\mathrm{DCA}]$ ions then con- 


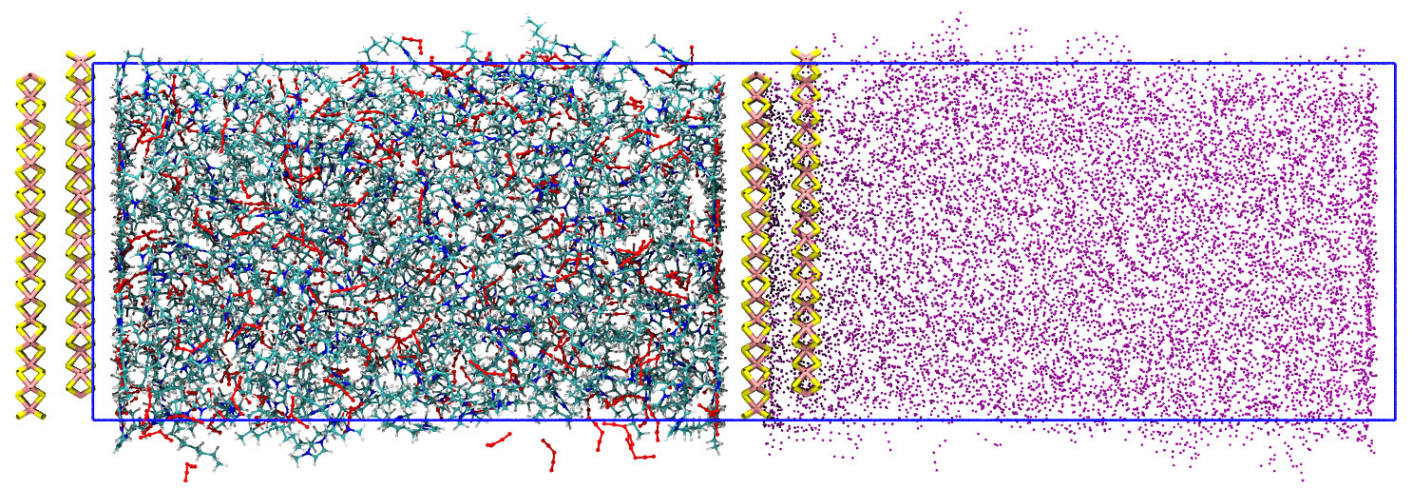

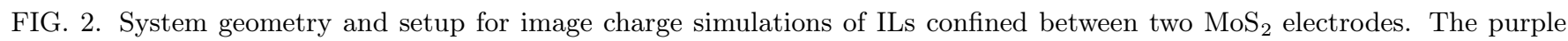
points are image charges. The blue box shows the periodic cell boundary. Each layer of $\mathrm{MoS}_{2}$ is covalently bonded to its images in the $x$ and $y$ directions.

centrate near the anode and cathode, respectively. However, the locations of the three interfacial layers are not affected.
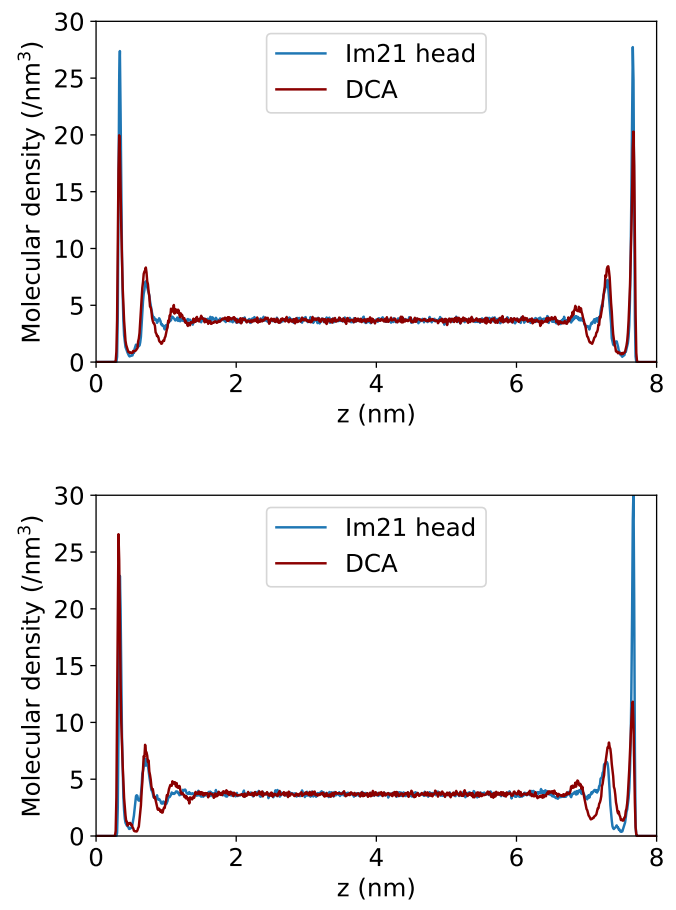

FIG. 3. Distributions of COM of different fragments along $z$ direction in the [Im21][DCA] system under $0 \mathrm{~V}$ (upper plot) and $2 \mathrm{~V}$ (lower plot).

The angles formed between ions and the plane of electrodes are analyzed to reveal the orientations of ions located in the first layer. Fig. 4 illustrates the definitions of three kinds of angles: the angle between the plane of an imidazolium ring and the electrode $(\alpha)$; the angle between the end-to-end vector of an octyl chain and the electrode $(\beta)$; and the angle between the plane of a [DCA] anion and the electrode $(\gamma)$. In [ImB1][DCA], the angle between the plane of benzyl ring and the electrode is also considered. The value of these angles range from $0^{\circ}$ to $90^{\circ}$. The former corresponds to a parallel orientation to the electrode and the latter to a perpendicular one.

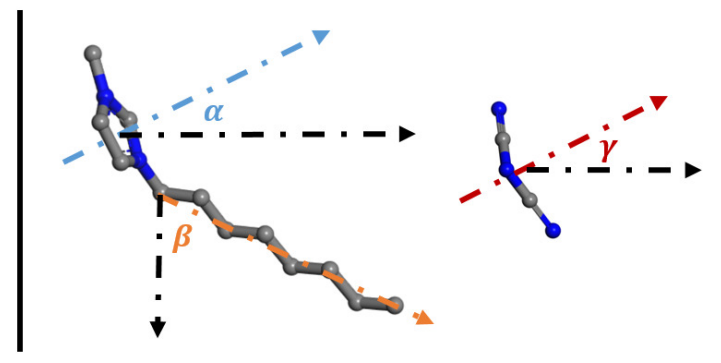

FIG. 4. Illustration of the angles formed between imidazolium head, octyl tail or [DCA] anion and the electrode. The vertical line at the left side represents the electrode in $x y$ plane. Hydrogen atoms are omitted for clarity.

The angle distributions for the [Im21][DCA] system is shown in Fig. 5. Both the imidazolium rings and the $[\mathrm{DCA}]$ anion tend to be oriented parallel to the electrode plane. The applied voltage drop of $2 \mathrm{~V}$ has almost no effect on the the orientations of ions at either the cathode or the anode.

Fig. 6 shows the distributions of $\mathrm{COM}$ of imidazolium head groups and octyl tails in [Im81], and COM of [DCA] along $z$ direction. When there is no applied voltage, three layers located at $0.34,0.71$ and $1.11 \mathrm{~nm}$ away from the electrodes can be identified, which are close to the locations of the interfacial layers in [Im21][DCA]. That is, the introduction of octyl tails does not alter the distributions of ions at the interface in the absence of voltage drop. A sharp peak at $0.34 \mathrm{~nm}$ in the distribution of octyl tails indicates that they tend to lay parallel to the interface in the first layer, similarly with the imidazolium rings. This agrees with previous simulations on EDLs formed by imidazolium based ILs with long alkyl tails. ${ }^{28}$ In the insets in Fig. 6 it is shown that the bulk region of [Im81][DCA] 

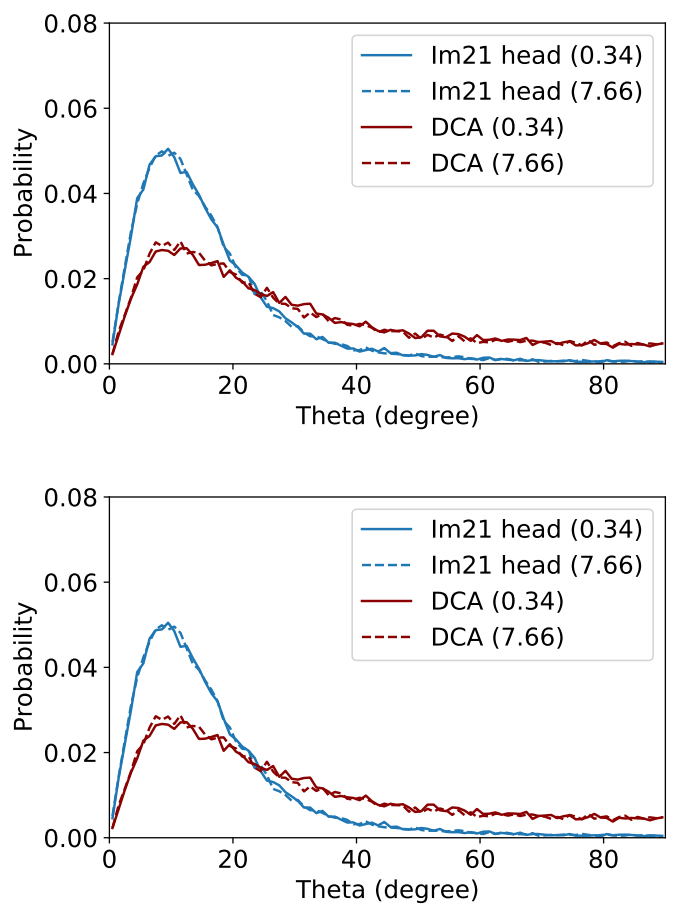

FIG. 5. Distributions of angles between imidazolium head groups or [DCA] and the $x y$ plane in the [Im21][DCA] system under $0 \mathrm{~V}$ (upper plot) and $2 \mathrm{~V}$ (lower plot). The number inside the bracket denotes the $z$ coordinate (in $\mathrm{nm}$ ) of corresponding peak in density distribution.

is not homogeneous. There are polar-group rich domains and non-polar group rich domains, alternatively. This nano-scale segregation into polar and non-polar domains is well documented for longer-chain ionic liquids. ${ }^{51} \mathrm{Be}-$ cause the distance between two electrodes is in the order of nanometers, a confinement effect might have a contribution to this heterogeneity. ${ }^{43}$ However, it should not have a pronounced effect on the structure of the EDLs. Because imidazolium rings and [DCA] always stay in the mutual vicinity in the bulk region, the polar and nonpolar segregation does not induce charge oscillations in bulk region. The applied voltage drop of $2 \mathrm{~V}$ promotes the enrichment of [Im81] and [DCA] near the anode and the cathode, respectively. The interfacial structure at the anode is similar to that at $0 \mathrm{~V}$, except for the intensity of each peak. At the cathode, however, significant structural changes are observed. The interface with the cathode can not be simply identified as three layers any more. The octyl tails are pushed away from the first layer, therefore forming a non-polar rich region at around $0.8 \mathrm{~nm}$ from the cathode, which in turn induces strong oscillation of polar and non-polar domains.

The distributions of angles formed between the imidazolium head group, the octyl tail in [Im81] cation or the $[\mathrm{DCA}]$ anion and the $x y$ plane are shown in Fig. 7 . Note that the tails analyzed in this figure are the octyl chains attached to the imidazolium rings located in the
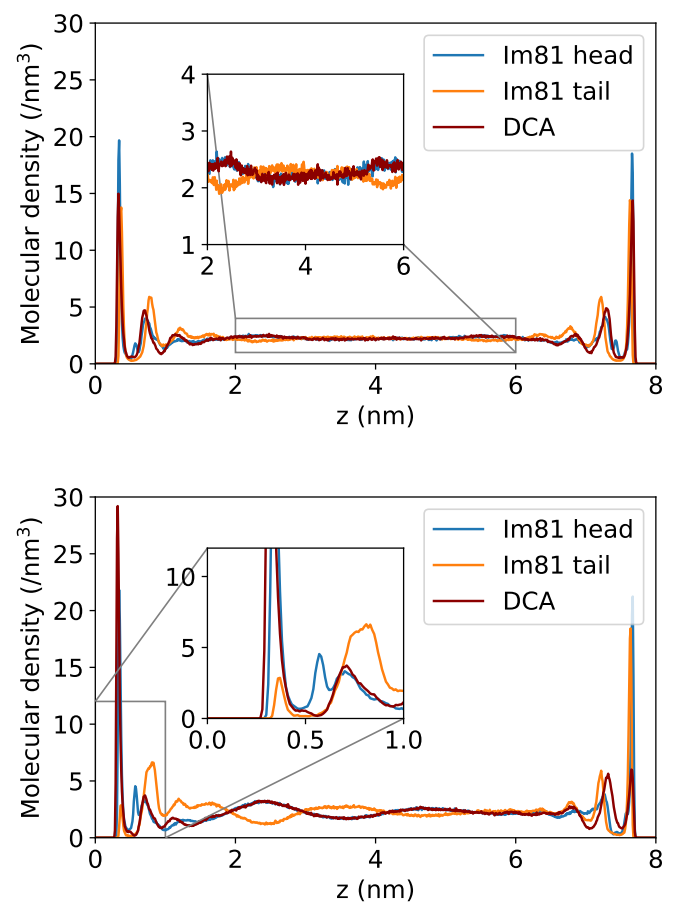

FIG. 6. Distributions of COM of different fragments along the $z$ direction in [Im81][DCA] system under $0 \mathrm{~V}$ (upper plot) and $2 \mathrm{~V}$ (lower plot).

first layer. Without voltage drop, both the imidazolium rings, the octyl tails and the [DCA] anions lay parallel to the electrode. The orientations of imidazolium head groups and [DCA] are almost the same as in [Im21]. The applied voltage drop of $2 \mathrm{~V}$ has almost no effect on the orientation of imidazolium head groups, while the [DCA] at the cathode orient slightly more parallel to the electrode than those at the anode, which can be attributed to the electrostatic attraction between the three negatively charged nitrogen atoms in $[\mathrm{DCA}]$ and the cathode. However, the orientation of octyl tails is significantly altered by the applied voltage. It is shown that octyl tails at the cathode are more likely to be perpendicular to the interface, while the tails at the anode are still preferentially parallel to the electrode. This orientational change is counter-intuitive at first sight. Considering the attraction between the anode and the imidazolium head groups, it would be expected that the octyl tail leave the first layer near the anode so that more space is available for imidazolium groups. The orientational change observed can be attributed to the more favorable interactions between counter-ions than the interaction between alkyl tails and [DCA] anions. At the cathode, [DCA] occupy the first layer, then either imidazolium rings or octyl tails will be excluded from the first layer. Here the more favorable interactions between oppositecharged ions results in the octyl chains being excluded. A recent simulation work on butyltrimethylammonium bis(trifluoromethanesulfonyl)amide at a graphene elec- 
trode revealed a similar scenario, with butyl tails more likely to be found perpendicular to the electrode at the cathode. ${ }^{31}$ It was shown that the turning point in voltage for the butyl tails to be more likely perpendicular to the electrode is $1 \mathrm{~V}$ at the cathode, whereas the corresponding value is $-5 \mathrm{~V}$ at anode.
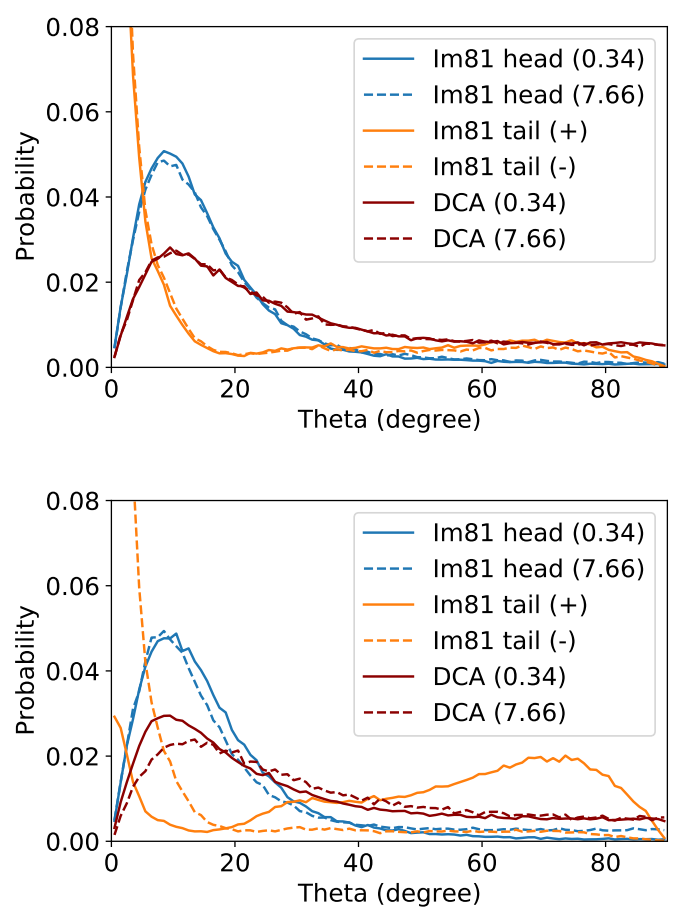

FIG. 7. Distributions of angles between imidazolium head groups, end-to-end vectors in octyl tails or [DCA] and $x y$ plane in the [Im81][DCA] system under $0 \mathrm{~V}$ (upper plot) and $2 \mathrm{~V}$ (lower plot). The number inside the bracket denotes the $z$ coordinate (in $\mathrm{nm}$ ) of corresponding peak in density distribution, while the + and - denote the octyl tails bonded to the imidazolium head groups near the cathode and anode, respectively.

Fig. 8 shows the distributions of COM of imidazolium head groups and benzyl side groups in [ImB1] ions, and $\mathrm{COM}$ of $[\mathrm{DCA}]$ ions, along the $z$ direction. In the absence of voltage drop, the first layer is mostly occupied by imidazolium rings and [DCA] anions. Only a small amount of benzyl groups are present in the first layer. The peak located at around $0.46 \mathrm{~nm}$ represents the benzyl groups that are attached to the imidazolium rings of the first layer. Also note that there is a peak for imidazolium head groups located at around $0.58 \mathrm{~nm}$, which represents mainly the imidazolium rings attached to the benzyl groups in the first layer.

From the orientational distributions as shown in Fig. 9, both the imidazolium and benzyl groups located at $0.34 \mathrm{~nm}$ are parallel to the electrode surface, while the imidazolium rings located at $0.58 \mathrm{~nm}$ and the benzyl groups located at $0.46 \mathrm{~nm}$ are perpendicular to the electrodes. An applied voltage drop of $2 \mathrm{~V}$ has no effect to the shape of the orientational distributions, even thought the intensities of the density peaks are affected.

The [ImB1] cation adopts two main conformations in the first interfacial layer, as shown in Fig. 10. The first consists of imidazolium rings parallel to the electrode, while the benzyl ring is perpendicular with one hydrogen atom of the benzyl ring pointing toward the electrode. The second conformation corresponds to the benzyl ring parallel to the electrode, while the imidazolium ring is perpendicular to the electrode, with the methyl group pointing to the bulk electrolyte. In the absence of a voltage drop, the first conformation is dominant. Applying of voltage drop of $2 \mathrm{~V}$ induces exchange between these two conformations. In the first layer near the cathode, most of the [ImB1] that adopted the first conformation reorient into the second, because of repulsion between cathode and positively-charged imidazolium head-groups. At anode, the second conformation disappears.
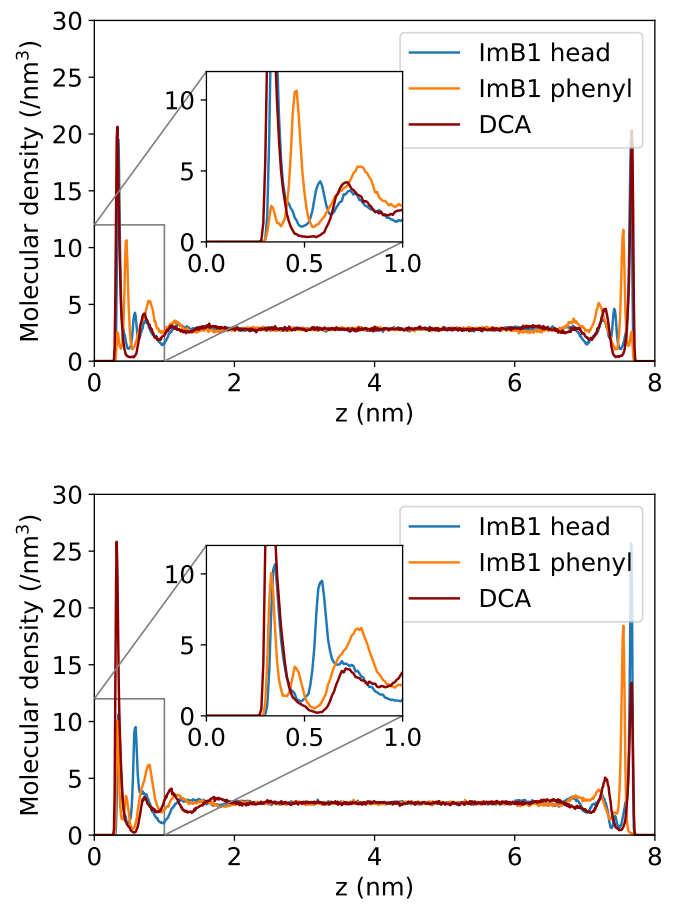

FIG. 8. Distributions of COM of different fragments along the $z$ direction in the $[\mathrm{ImB} 1][\mathrm{DCA}]$ system under $0 \mathrm{~V}$ (upper plot) and $2 \mathrm{~V}$ (lower plot).

Fig. 11 shows the distributions of COM of imidazolium rings and oxygen atoms in [ImO1] ions, and also COM of [DCA] ions, along $z$. The distributions of imidazolium rings and $[\mathrm{DCA}]$ anions are almost identical to those in [Im21][DCA]. The oxygen atoms of the hydroxyl groups are more likely to stay in the first layer near the cathode. However, because of its flexibility, the location of the hydroxyl group has a minor effect on the structural arrangement of imidazolium head groups and anions. The orientations of ions are also similar to those in $[\operatorname{Im} 21][\mathrm{DCA}]$, and thus are not shown here. 

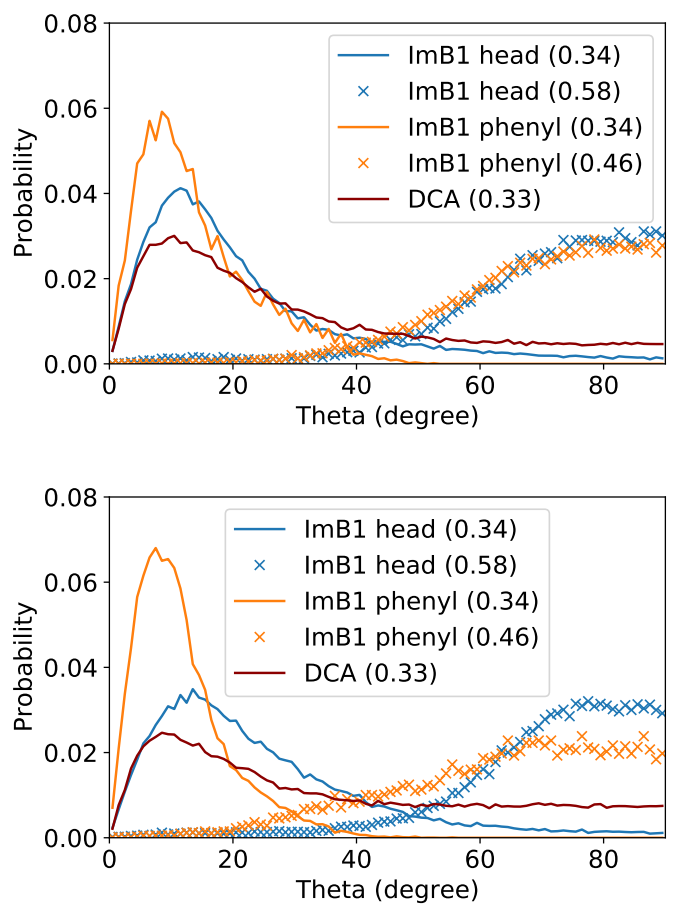

FIG. 9. Distributions of angles between imidazolium headgroups or benzyl rings or [DCA], and the $x y$ plane in the [ImB1][DCA] system under $0 \mathrm{~V}$ (upper plot) and $2 \mathrm{~V}$ (lower plot). The number inside the bracket denotes the $z$ coordinate (in $\mathrm{nm}$ ) of corresponding peak in density distribution. The profiles for ions near the anode are not shown for clarity.
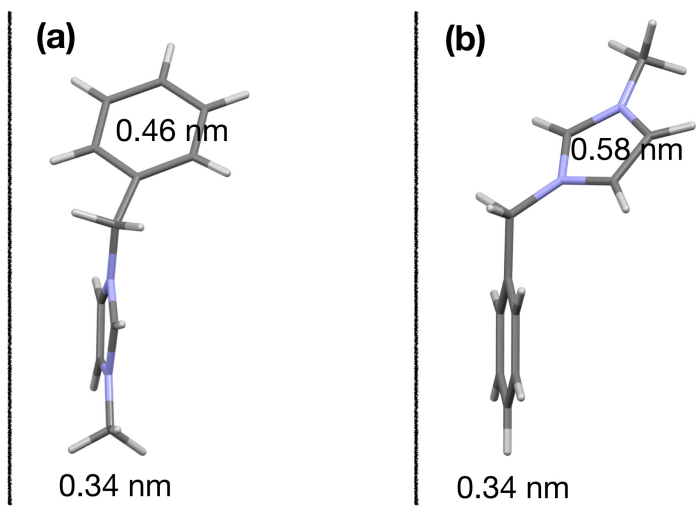

FIG. 10. The two main conformations of [ImB1] cations in the first layer. The vertical line in each sub-figure represents the electrode. The positions on the figure denotes the distances along $z$ between the COM of the imidazolium or of the benzyl ring and the electrode.

\section{B. Electrical properties of the EDLs}

We are particularly interested in the link between the interfacial ordering and the electrical properties. Herein, the voltage profiles are extracted from the simulation trajectories and enable evaluation of capacitance. The volt-
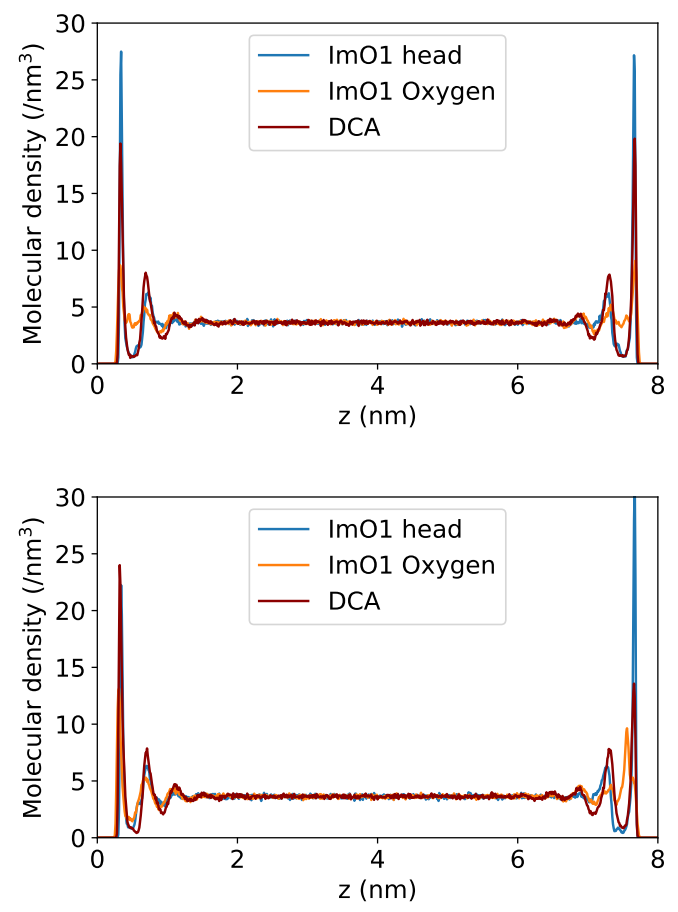

FIG. 11. Distributions of COM of different fragments along the $z$ direction in [ImO1][DCA] system under $0 \mathrm{~V}$ (upper plot) and $2 \mathrm{~V}$ (lower plot).

age profile along the $z$ direction can be calculated by double integration of the charge distribution of the electrolytes, $\rho(z)$, and of the charge density on the electrodes, $Q$, based on the one-dimensional Poisson equation:

$$
V(z)=-\frac{1}{\epsilon_{0}}\left(\iint \rho(z) \mathrm{d}^{2} z+Q d\right)
$$

where $\epsilon_{0}$ is the vacuum permittivity.

Fig. 12 shows the charge distributions in the electrolytes. On the anode side, the first peak of positive charge is $0.27 \mathrm{~nm}$ away from the electrode. This distance is the same for all four electrolytes, because it is mainly determined by the vdW repulsion between $\mathrm{S}$ atoms of the electrode and the imidazolium ring. On the cathode side, the first peak of negative charge is $0.29 \mathrm{~nm}$ away from the electrode. However, we notice that a peak of positive charge is present at $0.25 \mathrm{~nm}$ away from the cathode, which is mainly due to hydrogen atoms from imidazolium cations. Even though the first layer at the cathode is dominated by [DCA] anions, hydrogen atoms from cations can approach the cathode more closely because of their smaller vdW radius, compared to those of heavy atoms.

Unlike in the fluctuating charge method, ${ }^{52}$ the charges on the electrodes are not directly obtainable in the present 3D periodic image-charge method, because the electrode atoms carry no charge by themselves. The effects of electrode polarization are described instead by 


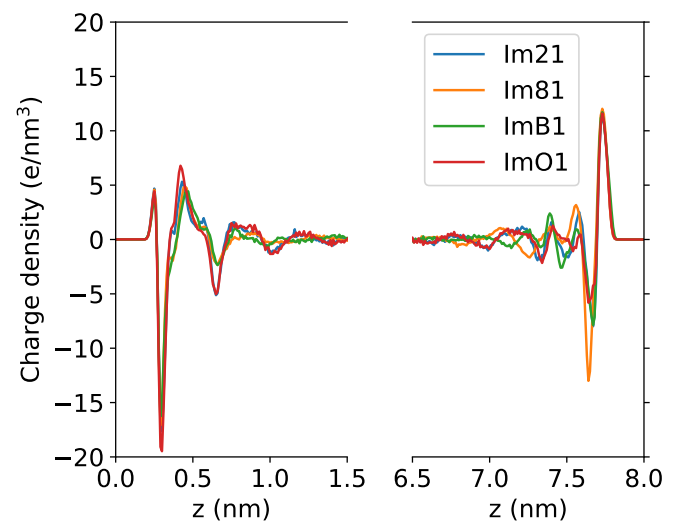

FIG. 12. Charge density distributions along the $z$ direction for different electrolytes under voltage drop of $2 \mathrm{~V}$.

the image charges and their infinite periodic images, together with the applied electric field. Herein, $Q$ is evaluated from the positions of electrolyte atoms, ${ }^{53}$

$$
Q=\sum_{i=1}^{n} \frac{q_{i} z_{i}}{d A}+\frac{\Delta V \epsilon_{0}}{d}
$$

where $q_{i}$ and $z_{i}$ are the charge and the $z$ coordinate of each electrolyte atom, and $A$ is the area of electrode.

Fig. 13 shows the voltage profiles along $z$ across the electrolyte region with different ILs. The computed voltage drops between the electrodes equals to the preset values, and this validates the image charge method. The voltage profiles reach a plateau in the bulk electrolyte beyond ca. $2 \mathrm{~nm}$ away from the electrodes, showing the strong electrostatic screening of the EDLs.

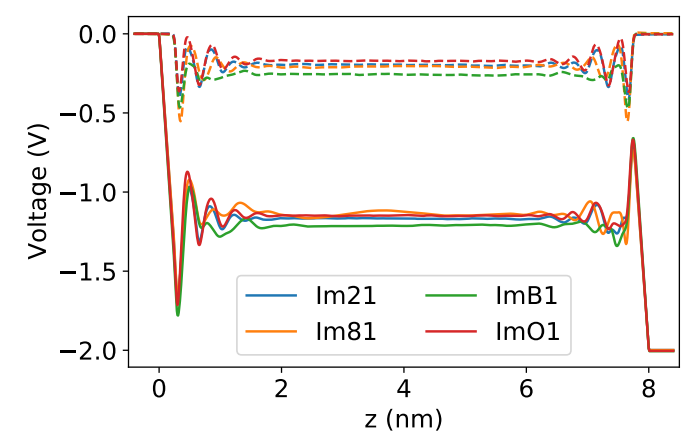

FIG. 13. Voltage profiles along the $z$ direction for different electrolytes under voltage drops of $0 \mathrm{~V}$ (dashed lines) and $2 \mathrm{~V}$ (solid lines).

From the calculated charge densities on the electrodes and the voltage profiles, the integral capacitances of the
EDLs at each electrode and the cell are evaluated as

$$
\begin{aligned}
C^{ \pm} & =\frac{Q}{V^{ \pm}-V_{0}^{ \pm}} \\
C^{\text {cell }} & =\frac{Q}{\Delta V}
\end{aligned}
$$

where $V^{ \pm}$is the voltage drop between each electrode and the bulk region, and $V_{0}^{ \pm}$is the value of $V^{ \pm}$in the absence of voltage drop between the electrodes. The calculated capacitances for different system are listed in Table II. For all ILs, the interfacial capacitance at the cathode is slightly larger than that at the anode. Among the four electrolytes, [ImB1][DCA] shows the largest cell capacitance, while [Im81][DCA] shows the smallest, the difference between them being quite small, only $8.5 \%$. The values of capacitance do not correlate with the molecular volumes of the ions, which indicates that the available space for counter-ions in the interfacial region is not the dominant factor for capacitance under voltage drop of $2 \mathrm{~V}$.

TABLE II. Calculated integral capacitances of EDLs under a voltage drop of $2 \mathrm{~V}$.

\begin{tabular}{cccc}
\hline Electrolyte & $C^{+}\left(\mathrm{\mu F} \mathrm{cm}^{-2}\right)$ & $C^{-}\left(\mathrm{\mu F} \mathrm{cm}^{-2}\right)$ & $C^{\text {cell }}\left(\mathrm{\mu F} \mathrm{cm}^{-2}\right)$ \\
\hline$[\mathrm{Im} 21][\mathrm{DCA}]$ & $4.89 \pm 0.008$ & $4.65 \pm 0.008$ & $2.38 \pm 0.004$ \\
{$[\mathrm{Im} 81][\mathrm{DCA}]$} & $5.08 \pm 0.025$ & $4.37 \pm 0.021$ & $2.35 \pm 0.012$ \\
{$[$ ImB1][DCA] } & $5.35 \pm 0.027$ & $4.87 \pm 0.025$ & $2.55 \pm 0.013$ \\
[ImO1][DCA] & $5.05 \pm 0.021$ & $4.83 \pm 0.021$ & $2.47 \pm 0.011$ \\
\hline
\end{tabular}

An applied voltage drop drives the cations and anions aggregating nearby anode and cathode, respectively. In order to inspect the charging mechanism, Fig. 14 shows the increase in the number of different species at the first layer near each electrode when voltage drop of $2 \mathrm{~V}$ is applied. An ion or fragment is considered to be located in the first layer if its COM is less than $0.5 \mathrm{~nm}$ away from the electrode.

In all of the systems, it is shown that the adsorption or desorption of [DCA] anions at the cathode or the anode dominates the behavior of ions at the interface, which can be attributed to the smaller size and therefore larger charge density of the [DCA] anion compared to imidazolium head-group. For [Im21], [ImB1] and [ImO1], the applied voltage drives imidazolium groups move into, or out of, the first layers of cathode or anode, which indicates a charing mechanism of exchange between counterions. However, the situation is different for [Im81]: at the cathode, the imidazolium head does not move out of the first layer. Instead, the tails are pushed out of the first layer, and more imidazolium head-groups enter into the first layer to occupy the created vacancies together with anions. At the anode, even though both imidazolium head groups and octyl chains become enriched in the first layer, the increment for octyl tails is larger than for imidazolium head-groups. Therefore, the charging mechanism for [Im81][DCA] is mainly the exchange between anions and octyl tails. 
Another phenomenon worth noting is that the increase in the number of imidazolium head-groups at the cathode, and its decrease at the anode, is more significant for [ImB1] than for the other cations. This can be attributed to the orientational preference of [ImB1] cation, as discussed in previous section. As shown in Fig. 10, the conformation (b) dominates the first layer at the cathode. The benzyl group tends to be aligned parallel to the electrode and drives the imidazolium ring out of the first layer. At anode the conformation (a) dominates, with the benzyl group tending to be placed perpendicular to the electrode and occupying less space. The presence of the benzyl group promotes the separation of imidazolium head-groups and [DCA] anions at the first interfacial layer. As a result, [ImB1][DCA] is able to deliver slightly larger capacitance than the other ILs.

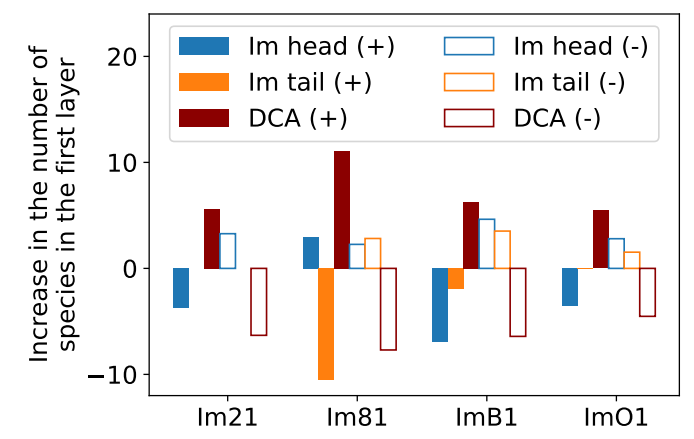

FIG. 14. The increase in the number of different species in the first layer near cathode (filled bars) or anode (unfilled bars) when voltage drop of $2 \mathrm{~V}$ is applied. The tail of [Im21] is not considered.

\section{Role of the alkyl tail of [Im81]}

It was recently reported that surface-active ILs promote enhanced capacitance by self-assembling into lamellar structures at the interface. ${ }^{26}$ In our simulation, even though polar and non-polar domain separation is observed in the bulk region, the [Im81] does not form pronounced layered structures near the anode. Instead, the octyl side chain is likely to occupy the first layer at the anode. Among the four ILs studied here, [Im81] delivers the smallest interfacial capacitance at the anode, as shown in Tab. II. It is therefore interesting to see whether the interfacial capacitance at the anode will increase if the octyl tails are forced to leave the first layer, so that more space is made available for imidazolium head-groups. We defined a reaction coordinate as the number of octyl tails in the first layer at the anode $\left(N_{\text {tail }}\right)$. In order to make $N_{\text {tail }}$ differentiable with respect to atomic positions, the contribution of an atom $i$ to its number density at position $\mathbf{r}$ is defined as a Gaussian function:

$$
\rho_{i}(\mathbf{r})=\frac{1}{\sigma \sqrt{2 \pi}} \exp \left(\frac{-\left|\mathbf{r}-\mathbf{r}_{i}\right|^{2}}{2 \sigma^{2}}\right)
$$

where $\mathbf{r}_{i}$ is the position of atom $i$, and $\sigma$ determines the radius of the atom, which we set to $0.05 \mathrm{~nm}$. Then $N_{\text {tail }}$ is evaluated as

$$
N_{\text {tail }}=\frac{1}{2} \sum_{i}^{\mathrm{C} 3-\mathrm{C} 8} \frac{1}{6} \operatorname{erfc}\left(\frac{z_{0}-z_{i}}{\sqrt{2} \sigma}\right)
$$

where C3-C8 means the third to eighth carbon atoms in the octyl tail, $z_{i}$ is the $z$ coordinate of each atom, and $z_{0}$ is the lower bound of the first layer at the anode, which we set to $7.5 \mathrm{~nm}$ based on the molecular distributions discussed in section III A. In order to force the octyl tail to leave the first layer, a harmonic biasing potential is applied to restrain the $N_{\text {tail }}$ to a target value:

$$
U_{\text {umbrella }}=\frac{1}{2} k\left(N_{\text {tail }}-N_{\text {target }}\right)^{2}
$$

where $k$ is the harmonic force constant, which we set to $100.0 \mathrm{~kJ} \mathrm{~mol}^{-1}$. Three simulations were performed with $N_{\text {target }}$ set to $0.0,10.0$ and 20.0, under the voltage drop of $2 \mathrm{~V}$.

Fig. 15 shows the number of imidazolium head-groups and [DCA] anions located in the first layer of the anode, when different $N_{\text {target }}$ are applied. The integral capacitance of the cell is also plotted. When $N_{\text {target }}$ is decreased, the octyl tails are forced to leave the first layer. As expected, more imidazolium head-groups are then able to occupy the first layer because of the free space created. However, it is found that the number of [DCA] ions in the first layer increases almost in parallel with the number of imidazolium groups. This indicates that the imidazolium groups and the [DCA] anions move into or out of the first layer in a correlated manner. Therefore, the charge that can be stored in the interface is not affected, which results in an essentially similar capacitance independently of the value of $N_{\text {target }}$.

\section{Charging performance}

The speed of charge accumulation on the electrodes is one of the key properties of EDL devices, since it determines the power of EDLC and the gating speed of the FET. In order to inspect the charging process of the cell, initial configurations were taken from the system equilibrated under $0 \mathrm{~V}$. Then a voltage drop of $2 \mathrm{~V}$ was applied to the electrodes and the evolution of charge density accumulated on the electrodes was extracted according to equation (5).

Fig. 16 shows the charge density on the electrodes plotted against time, averaged from three trajectories started from different initial configurations. It is shown that the [Im21][DCA] displays the fastest charging. The charging 


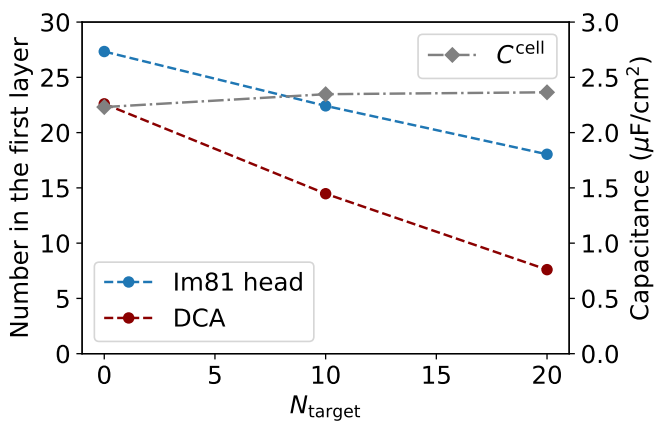

FIG. 15. Number of imidazolium head-groups and [DCA] anions in the first layer near the anode, and integral capacitance of the cell at different settings of $N_{\text {target }}$ for [Im81][DCA], under a voltage drop of $2 \mathrm{~V}$.

performance for [ImO1] is close to that for [Im21] and is significantly better than those for [ImB1] and [Im81]. These results are surprising given that [ImO1] is three times more viscous than [Im21] and has comparable viscosity to [ImB1] and [Im81]. As such, we conclude that the charging performance is not strongly correlated with macroscopic dynamics. We interpret this results as being due to the charging processes of [Im21] involving only the displacement of small ions in adjacent layers, whereas that for [Im81] and [ImB1] are accompanied by conformational changes of cations at the interface. During the charging process, the octyl tails of [Im81] near the cathode are pushed away from the first layer and reorient to become perpendicular to the interface. For [ImB1], however, most of the cations of the first layer near the cathode change their conformations from (a) to (b), as shown in Fig. 10. These local conformational change slow down the charging of the EDL. In [ImO1], the 2-hydroxyethyl groups also change their orientations near anode, but the barrier for this conformational change is low because of the small size and flexibility. Therefore, the charging performance of [ImO1] is close to that of [Im21] and much higher than those of [Im81] and [ImB1], even though [ImO1] is much more viscous than [Im21]. From our simulations, the full charging of the cells happens at the time scale of nanoseconds. It is much shorter than experimental charing times reported for a IL-EDLC ${ }^{54}$ and a IL-FET, ${ }^{55}$ which are in the order of seconds. The discrepancy can be attributes to two factors: Firstly, in both the EDLC and FET mentioned above, porous electrodes are used, in which the diffusion of ions into or out of the electrodes dominates the charging speed; Secondly, the simulations were performed at $333 \mathrm{~K}$ in this work, which allows for much faster dynamics than at ambient temperature.

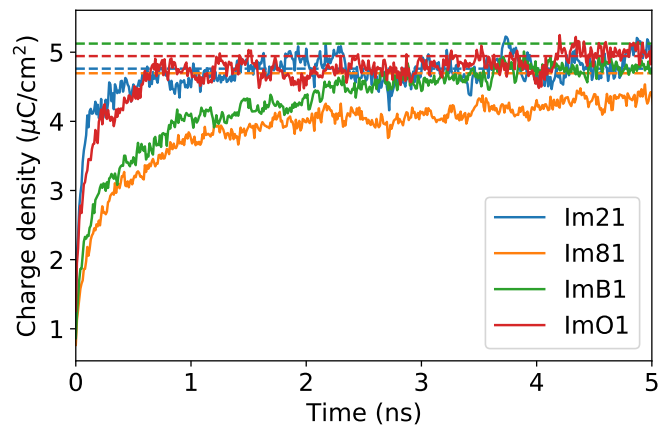

FIG. 16. Time evolution of the charge densities accumulated at the electrodes for different electrolytes when voltage drop of $2 \mathrm{~V}$ is applied. The horizontal dashed lines denote the converged values of charge densities.

\section{CONCLUSIONS}

We have performed polarizable MD simulations to study the interfaces between four imidazolium dicyanamide ionic liquid electrolytes and $\mathrm{MoS}_{2}$ electrodes. The effect of side chain modifications to the structural, electrical and dynamic properties of the electric double layers are studied by using ethyl, octyl, benzyl and 2-hydroxyethyl side groups on the imidazolium cations ([Im21], [Im81], [ImB1] and [ImO1], respectively).

The octyl and benzyl groups are found to have prominent effects on the interfacial structure, while the 2hydroxyethyl group has almost no effect. In the absence of a voltage drop, the octyl tails of the [Im81] cation tend to lay parallel to the electrode in the first interfacial layer. An applied positive voltage drives the octyl tails out from the first layer, and they become perpendicular to the electrode, whereas an applied negative voltage has minor effect on the orientation of the octyl chains. The [ImB1] cation displays two major conformations in the first layer of the interface, one with the imidazolium head-group parallel to the electrode and the other with the benzyl group parallel to the electrode. In the absence of a voltage drop, the first conformation dominates. An applied positive voltage promotes a conformational change from the first to the second one.

The difference in interfacial structures results in distinct charging mechanisms. The charging mechanisms for [Im21], [ImB1] and [ImO1] consist mainly in exchange between counter-ions, whereas the mechanism for [Im81] is mainly the exchange between [DCA] anions and octyl tails. The separation between counter-ions is more pronounced for $[\mathrm{ImB} 1][\mathrm{DCA}]$ than for other ILs. This is due to the orientational preference of the [ImB1] cation. At cathode, the benzyl group lays parallel to the electrode, driving imidazolium group away from the first layer. While at anode, the benzyl group prefers to be perpendicular to the electrode, allowing imidazolium group to enter the first layer more easily. The benzyl group in 
[ImB1] assists with the separation of imidazolium group and [DCA] anion, therefore allows [ImB1][DCA] to deliver slightly larger capacitance.

In spite of the difference in molecular volumes and interfacial structures, all the four ILs deliver similar capacitances. It is found that the space available at the interface has little effect on the capacitance under a voltage drop of $2 \mathrm{~V}$. Instead, the capacitance is mainly determined by the association between counter-ions. In [Im81], even when the octyl tails are forced out of the first layer, the space s created at the interface are occupied by neutral clusters of imidazolium head-groups and [DCA] anions, and as such the capacitance is not sharply affected. This result is comparable to the observation that the addition of organic solvents does not have significant effect on the interfacial capacitance, because of the balance between ionic concentration and the separability of the counter-ions. ${ }^{16}$

From the analysis of charge accumulation on the electrodes during charging, it is shown that the charging speed with [ImO1] is close to that of [Im21], and is significantly larger than those of [Im81] and [ImB1], even thought the viscosity of [ImO1] is closer to that of [Im81] and [ImB1], than to [Im21], which is considerably lower. This difference in charging is attributed to the slow conformational changes of [Im81] and [ImB1] near the cathode during charging, and indicates that the charging performance does not correlate strongly with macroscopic dynamics. Instead, it is mainly determined by local reorientations and displacements at the interface.

This work emphasizes the importance of how microscopic molecular structures of ILs affect the properties of EDLs. In order to optimize the interfacial capacitance at a planar electrode, efforts should be undertaken on how to effectively suppress the association between counterions at the interface. In contrast, the capacitance is largely insensitive to the molecular volume for ILs of the same family. The present results also point out that bulk dynamic properties like viscosity do not determine by themselves the charging performance of EDLs. Instead, the microscopic conformational changes happening at the interface assume an important role in the charging process, and should be taken into account in the rational design of IL electrolytes. In the present, all cations share the same imidazolium head. It is therefore intriguing to see whether these conclusions still stand for ILs belonging to different families (e.g. tetraalkylammonium, phosphonium), considering that the association strength between counter-ions can be very different.

\section{FORCE FIELD AND SIMULATION DETAILS}

The van der Waals (vdW) parameters for $\mathrm{MoS}_{2}$ were obtained from the work of Sresht et. al. ${ }^{56}$ Since the image-charge method is used to describe the Coulomb interactions between $\mathrm{MoS}_{2}$ and the electrolytes, the electrode atoms carry no charge. The recently-developed
CL\&Pol ${ }^{46,50}$ polarizable force field is used to describe the ILs. In order to avoid the polarization catastrophe, the electrostatic interactions between hydrogen atoms in hydroxyl group and all Drude dipoles are damped by using a Tang-Tonnies function. ${ }^{57}$ The Thole screening parameter and non-bonded parameters for hydroxyl and benzyl group are refined to provide better agreement with experimental viscosity data.

The CL\&Pol model was developed on top of the nonpolarizable CL\&P force field ${ }^{58}$ by introducing Drude induced dipoles to heavy atoms. After the introduction of Drude induced dipoles, the Lennard-Jones (LJ) parameters are scaled, because the non-polarizable force field had been optimized to account for part of the polarization implicitly. A predictive scheme to evaluate these scaling factors for LJ well-depths from the charge and dipole moment of fragments was proposed during the development of CL\&Pol. ${ }^{46}$ The scaling factor for the LJ radius is empirically determined to be 0.985 based on the calculated densities.

The refined force field for ILs was validated by calculating the viscosity and density of of several ILs at $333 \mathrm{~K}$ and 1 bar. The viscosities are calculated with periodic perturbation method, ${ }^{59}$ which has proven to be accurate and efficient for viscous liquids like ionic liquids, or liquids under extreme pressure ${ }^{60,61}$ In this method, an acceleration along the $x$ direction, with periodicity in $z$, is applied in an otherwise normal $N p T$ simulation:

$$
a_{x}(z)=A \cos \left(\frac{2 \pi z}{l_{z}}\right)
$$

where $l_{z}$ is the $z$-length of the simulation box. In steady state, the acceleration will generate a velocity gradient:

$$
v_{x}(z)=W \cos \left(\frac{2 \pi z}{l_{z}}\right)
$$

from which the velocity amplitude $W$ can be calculated by ensemble averaging of the simulation data. Via Stokes' equation, the amplitude is related to the viscosity $\eta$ by:

$$
W=\frac{A \rho}{\eta}\left(\frac{l_{z}}{2 \pi}\right)^{2}
$$

Viscosities were calculated at four accelerations (0.01, $0.02,0.03$ and $0.04 \mathrm{~mm} \mathrm{ps}^{-2}$ ), from which a linear extrapolation towards zero acceleration is performed to obtain the zero-shear viscosity. ${ }^{60,62}$

The density and viscosity are calculated with different values of the Thole parameter to determine its effect. As shown in Fig. 17, the Thole parameter has little effect on these properties, with the exception that the density calculated with extended-Langrangian (EL) approach is smaller than the result of self-consistent field (SCF) method when the Thole parameter is set to the usual value of 2.6. By inspecting the root-mean-squared force (RMSF) on Drude particles after $20 \mathrm{SCF}$ iterations 
at the last step, it is shown that decreasing the Thole parameter makes the SCF iterations more robust. Therefore, the Thole parameter is chosen to be 1.8 in this work to enhance the stability of the Drude model without affecting the thermodynamic and transport properties.
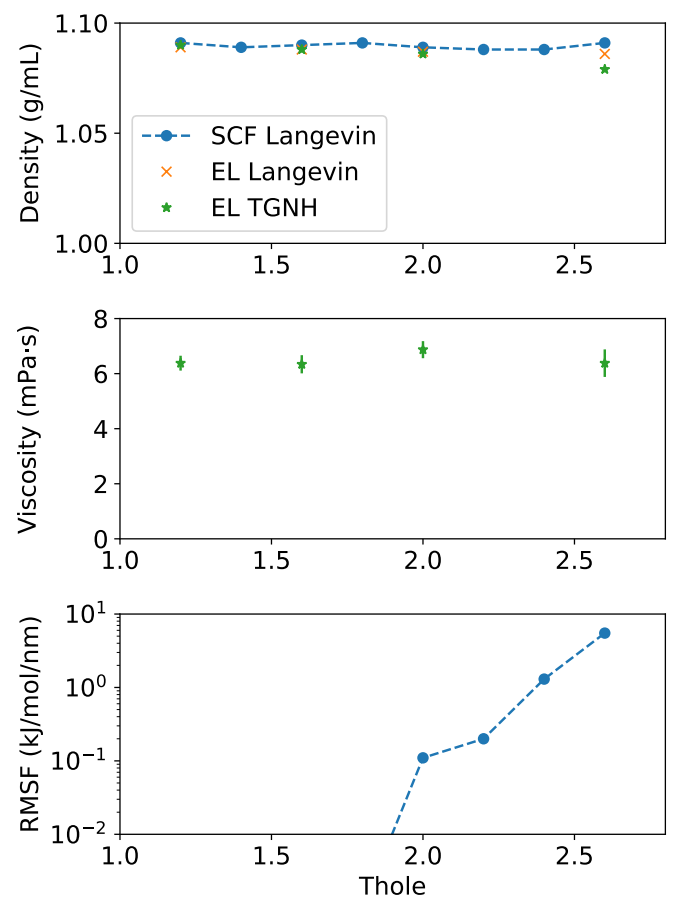

FIG. 17. Calculated density, viscosity, and the RMSF on Drude particles for bulk [Im21][DCA] at $333 \mathrm{~K}$ with different values of the Thole parameter. The uncertainty of density is too small to be shown.

In the CL\&Pol force field, the LJ well-depth between cation-anion, cation-neutral, anion-neutral and neutralneutral fragments are scaled down using the predictive scheme, ${ }^{46}$ whereas the LJ well-depth between fragments with charges of the same sign were kept untouched (since these are seldom in the respective vicinity). In this work, the LJ well-depth between fragments with charges of the same sign are also scaled down based on the predictive scheme. ${ }^{46}$ However, the predictive scheme requires a minimal-energy distance between fragments optimized by QM calculation, which is not applicable for same-charge fragments. As such, we estimate the distance between same-charge fragments by

$$
\begin{array}{r}
d(\text { frag } 1, \text { frag } 2)=d(\text { frag } 1, \text { butane })+d(\text { frag } 2, \text { butane }) \\
-d(\text { butane, butane })
\end{array}
$$

After scaling down the LJ well-depth of same-charge fragments, the experimental viscosities for alkylimidazolium dicyanamide were more accurately reproduced, as shown in Table III.

The Drude polarizable trajectories were generated with an integrator using the extended-Langrangian approach, where the COM motion of real atoms and attached Drude particles are thermalized at $333 \mathrm{~K}$, unless otherwise specified, while the relative motion between atoms and their Drude particles are thermalized at $1 \mathrm{~K}$ to approximate the SCF relaxation of Drude dipoles. ${ }^{63}$ The electrode atoms are thermalized using a Langevin thermostat with a friction coefficient of $5 \mathrm{ps}^{-1}$. The IL atoms are thermalized using the recently developed temperature-grouped Nosé-Hoover (TGNH) thermostat in order to attain more reliable dynamic properties. ${ }^{64}$ With the conventional dual-Nosé-Hoover (NH) thermostat, ${ }^{63,65}$ the temperatures of different degrees of freedoms (DOFs) cannot be properly maintained. Because of the continuous heat flow from DOFs of real atoms to Drude particles, the temperature of molecular COM motions becomes significantly higher than that of other DOFs, which causes underestimation of density and overestimation of diffusion. As a remedy, the TGNH thermostat thermalizes the DOFs of molecular COM motions, atomic internal motions relative to molecular COM and Drude relative motions separately, which gives more accurate estimations of density and diffusion coefficient. ${ }^{64}$ Three Nosé-Hoover chains were used for the TGNH thermostat. The coupling strength for real-atom DOFs and Drude DOFs are $10 \mathrm{ps}^{-1}$ and $40 \mathrm{ps}^{-1}$, respectively. As shown in Table III, the conventional NH thermostat is not able to correctly thermalize Drude-polarizable systems. The temperature of molecular COM motion is over $50 \mathrm{~K}$ higher than the preset value, which results in significant underestimation of viscosity and density. In contrast, the TGNH thermostat is able to maintain the average molecular translational temperature, thus predicting density to be the same as the one obtained with the Langevin thermostat.

The vdW and the real space electrostatic interactions are truncated at $1.2 \mathrm{~nm}$. The particle mesh Ewald $\operatorname{method}^{70}$ is applied to handle the long-range electrostatic interactions in reciprocal space. The integration time step is $1 \mathrm{fs}$. All bonds involving hydrogen and the $\mathrm{C}-\mathrm{O}-\mathrm{H}$ angles in [ImO1] are constrained by using SHAKE algorithm. ${ }^{71}$ In $N p T$ simulations for determining density, viscosity and permittivity, a Monte Carlo barostat $^{72,73}$ was used to maintain pressure, wherein the volume of simulation box and positions of molecular centers were scaled every 100 ps. The uncertainties reported in this work were estimated as the standard errors of five blocks using the block-average method. The extendedLangrangian simulations were performed with OpenMM 7.4.1, ${ }^{45,74}$ while the SCF simulations were performed using GROMACS 2016. ${ }^{75}$ OpenMM is extensively accelerated on GPU processors, allowing sufficiently long simulation trajectories to be generated. The methods used in this work were implemented as an OpenMM plug-in. The source code is hosted at https://github.com/z-gong/ openmm-velocityVerlet. 
TABLE III. Calculated viscosity, density and molecular COM temperature for several ILs with the refined CL\&Pol force field using different thermostats. The uncertainties for calculated density are smaller than $0.001 \mathrm{~g} \mathrm{~cm}^{-3}$. The experimental data ${ }^{66-69}$ are listed in the brackets for comparison.

\begin{tabular}{|c|c|c|c|c|c|}
\hline Ionic liquid & Thermostat & $T(\mathrm{~K})$ & $T_{\mathrm{COM}}(\mathrm{K})$ & Viscosity (mPas) & Density $\left(\mathrm{g} \mathrm{cm}^{-3}\right)$ \\
\hline \multirow[t]{3}{*}[\operatorname{Im}21]{$[\mathrm{DCA}]$} & TGNH & 333 & $333.3 \pm 0.4$ & $6.3 \pm 0.3(6.95)$ & $1.088(1.081)$ \\
\hline & Langevin & 333 & $334.9 \pm 0.9$ & - & $1.088(1.081)$ \\
\hline & $\mathrm{NH}$ & 333 & $388.8 \pm 1.3$ & $2.6 \pm 0.1(6.95)$ & $1.046(1.081)$ \\
\hline [Im61][DCA] & TGNH & 333 & $334.5 \pm 0.3$ & $17.0 \pm 1.1(15.0)$ & $0.996(1.008)$ \\
\hline [Im81][DCA] & TGNH & 333 & $332.7 \pm 0.2$ & $25.5 \pm 1.9$ & 0.976 \\
\hline \multirow[t]{2}{*}{ [ImB1][DCA] } & TGNH & 333 & $333.7 \pm 0.5$ & $26.5 \pm 2.3$ & 1.126 \\
\hline & Langevin & 298 & $297.9 \pm 0.9$ & - & $1.157(1.158)$ \\
\hline [ImO1][DCA] & TGNH & 333 & $333.4 \pm 0.3$ & $19.6 \pm 1.2(20.7)$ & $1.166(1.164)$ \\
\hline
\end{tabular}

\section{DATA SHARING}

The computational tools and data that support the findings are made public in the above-mentioned repositories and are available from the corresponding author upon reasonable request.

\section{ACKNOWLEDGMENTS}

This work was supported through funding from the Agence Nationale de la Recherche (grant ANR-18-CE090018). A CC BY or equivalent licence is applied to Author Accepted Manuscript arising from this submission, in accordance with the grant's open access conditions. Computational resources were provided by IDRIS (national computing center of the CNRS) and the PSMN (computing center at ENS de Lyon).

${ }^{1}$ A. K. Geim and K. S. Novoselov, "The rise of graphene," 6, 183-191.

${ }^{2}$ C. Tan, X. Cao, X.-J. Wu, Q. He, J. Yang, X. Zhang, J. Chen, W. Zhao, S. Han, G.-H. Nam, M. Sindoro, and H. Zhang, "Recent advances in ultrathin two-dimensional nanomaterials," 117, 6225-6331, publisher: American Chemical Society.

${ }^{3}$ M. Watanabe, M. L. Thomas, S. Zhang, K. Ueno, T. Yasuda, and K. Dokko, "Application of Ionic Liquids to Energy Storage and Conversion Materials and Devices," Chemical Reviews 117, 7190-7239 (2017), publisher: American Chemical Society.

${ }^{4}$ D. R. MacFarlane, N. Tachikawa, M. Forsyth, J. M. Pringle, P. C. Howlett, G. D. Elliott, J. H. Davis, M. Watanabe, P. Simon, and C. A. Angell, "Energy applications of ionic liquids," Energy \& Environmental Science 7, 232-250 (2014), publisher: The Royal Society of Chemistry.

${ }^{5}$ M. Armand, F. Endres, D. R. MacFarlane, H. Ohno, and B. Scrosati, "Ionic-liquid materials for the electrochemical challenges of the future," Nature Materials 8, 621-629 (2009).

${ }^{6}$ T. Fujimoto and K. Awaga, "Electric-double-layer field-effect transistors with ionic liquids," Physical Chemistry Chemical Physics 15, 8983-9006 (2013), publisher: The Royal Society of Chemistry.

${ }^{7}$ F. Béguin, V. Presser, A. Balducci, and E. Frackowiak, "Carbons and Electrolytes for Advanced Supercapacitors," Advanced Materials 26, 2219-2251 (2014), _eprint: https://onlinelibrary.wiley.com/doi/pdf/10.1002/adma.201304137. ${ }^{8}$ S. Baldelli, "Surface Structure at the Ionic Liquid-Electrified Metal Interface," Accounts of Chemical Research 41, 421-431 (2008), publisher: American Chemical Society.
${ }^{9}$ M. V. Fedorov and A. A. Kornyshev, "Ionic Liquids at Electrified Interfaces," Chemical Reviews 114, 2978-3036 (2014), publisher: American Chemical Society.

${ }^{10} \mathrm{~J}$. Vatamanu, O. Borodin, M. Olguin, G. Yushin, and D. Bedrov, "Charge storage at the nanoscale: understanding the trends from the molecular scale perspective," Journal of Materials Chemistry A 5, 21049-21076 (2017), publisher: The Royal Society of Chemistry.

${ }^{11}$ R. Burt, G. Birkett, and X. S. Zhao, "A review of molecular modelling of electric double layer capacitors," Physical Chemistry Chemical Physics 16, 6519-6538 (2014), publisher: The Royal Society of Chemistry.

${ }^{12}$ C. Merlet, B. Rotenberg, P. A. Madden, and M. Salanne, "Computer simulations of ionic liquids at electrochemical interfaces," Physical Chemistry Chemical Physics 15, 15781-15792 (2013).

${ }^{13} \mathrm{~J}$. Vatamanu, O. Borodin, and G. D. Smith, "Molecular Simulations of the Electric Double Layer Structure, Differential Capacitance, and Charging Kinetics for N-Methyl-Npropylpyrrolidinium Bis(fluorosulfonyl)imide at Graphite Electrodes," The Journal of Physical Chemistry B 115, 3073-3084 (2011), publisher: American Chemical Society.

${ }^{14}$ C. Merlet, B. Rotenberg, P. A. Madden, P.-L. Taberna, P. Simon, Y. Gogotsi, and M. Salanne, "On the molecular origin of supercapacitance in nanoporous carbon electrodes," Nature Materials 11, 306 (2012), publisher: Nature Publishing Group.

${ }^{15}$ C. Péan, C. Merlet, B. Rotenberg, P. A. Madden, P.-L. Taberna, B. Daffos, M. Salanne, and P. Simon, "On the Dynamics of Charging in Nanoporous Carbon-Based Supercapacitors," ACS Nano 8, 1576-1583 (2014), publisher: American Chemical Society.

${ }^{16}$ R. Burt, K. Breitsprecher, B. Daffos, P.-L. Taberna, P. Simon, G. Birkett, X. S. Zhao, C. Holm, and M. Salanne, "Capacitance of Nanoporous Carbon-Based Supercapacitors Is a Trade-Off between the Concentration and the Separability of the Ions," The Journal of Physical Chemistry Letters 7, 4015-4021 (2016), publisher: American Chemical Society.

${ }^{17}$ C. Pean, B. Rotenberg, P. Simon, and M. Salanne, "Understanding the different (dis)charging steps of supercapacitors: influence of potential and solvation," Electrochimica Acta 206, 504-512 (2016).

${ }^{18} \mathrm{~S} . \mathrm{Li}, \mathrm{Z}$. Zhao, and X. Liu, "Electric double layer structure and capacitance of imidazolium-based ionic liquids with FSI- and Tf- anions at graphite electrode by molecular dynamic simulations," Journal of Electroanalytical Chemistry , 113452 (2019), publisher: Elsevier BV.

${ }^{19}$ G. Feng, R. Qiao, J. Huang, S. Dai, B. G. Sumpter, and V. Meunier, "The importance of ion size and electrode curvature on electrical double layers in ionic liquids," Physical Chemistry Chemical Physics 13, 1152-1161 (2010), publisher: The Royal Society of Chemistry.

${ }^{20}$ G. Feng, S. Li, J. S. Atchison, V. Presser, and P. T. Cummings, "Molecular Insights into Carbon Nanotube Supercapacitors: Capacitance Independent of Voltage and Temperature," The Jour- 
nal of Physical Chemistry C 117, 9178-9186 (2013), publisher: American Chemical Society.

${ }^{21}$ J. Vatamanu, L. Cao, O. Borodin, D. Bedrov, and G. D. Smith, "On the Influence of Surface Topography on the Electric Double Layer Structure and Differential Capacitance of Graphite/Ionic Liquid Interfaces," The Journal of Physical Chemistry Letters 2, 2267-2272 (2011), publisher: American Chemical Society.

${ }^{22}$ J. Vatamanu, Z. Hu, D. Bedrov, C. Perez, and Y. Gogotsi, "Increasing Energy Storage in Electrochemical Capacitors with Ionic Liquid Electrolytes and Nanostructured Carbon Electrodes," The Journal of Physical Chemistry Letters 4, 2829-2837 (2013), publisher: American Chemical Society.

${ }^{23}$ M. P. S. Mousavi, B. E. Wilson, S. Kashefolgheta, E. L. Anderson, S. He, P. Bühlmann, and A. Stein, "Ionic Liquids as Electrolytes for Electrochemical Double-Layer Capacitors: Structures that Optimize Specific Energy," ACS Applied Materials \& Interfaces 8, 3396-3406 (2016), publisher: American Chemical Society.

${ }^{24}$ V. Lockett, R. Sedev, J. Ralston, M. Horne, and T. Rodopoulos, "Differential Capacitance of the Electrical Double Layer in Imidazolium-Based Ionic Liquids: Influence of Potential, Cation Size, and Temperature," The Journal of Physical Chemistry C 112, 7486-7495 (2008), publisher: American Chemical Society.

${ }^{25}$ S. Jo, S.-W. Park, Y. Shim, and Y. Jung, "Effects of Alkyl Chain Length on Interfacial Structure and Differential Capacitance in Graphene Supercapacitors: A Molecular Dynamics Simulation Study," Electrochimica Acta 247, 634-645 (2017).

${ }^{26}$ X. Mao, P. Brown, C. Červinka, G. Hazell, H. Li, Y. Ren, D. Chen, R. Atkin, J. Eastoe, I. Grillo, A. A. Padua, M. F. Costa Gomes, and T. A. Hatton, "Self-assembled nanostructures in ionic liquids facilitate charge storage at electrified interfaces," Nature Materials (2019), 10.1038/s41563-019-0449-6.

${ }^{27}$ M. V. Fedorov, N. Georgi, and A. A. Kornyshev, "Double layer in ionic liquids: The nature of the camel shape of capacitance," Electrochemistry Communications 12, 296-299 (2010).

${ }^{28}$ S. Wang, S. Li, Z. Cao, and T. Yan, "Molecular Dynamic Simulations of Ionic Liquids at Graphite Surface," The Journal of Physical Chemistry C 114, 990-995 (2010), publisher: American Chemical Society.

${ }^{29}$ Y. Su, J. Yan, M. Li, M. Zhang, and B. Mao, "Electric Double Layer of $\mathrm{Au}(100)$ /Imidazolium-Based Ionic Liquids Interface: Effect of Cation Size," The Journal of Physical Chemistry C 117, 205-212 (2013), publisher: American Chemical Society.

${ }^{30}$ A. Gallegos, C. Lian, B. Dyatkin, and J. Wu, "Sidechain effects on the capacitive behaviour of ionic liquids in microporous electrodes," Molecular Physics 117, 3603-3613 (2019), publisher: Taylor \& Francis eprint: https://doi.org/10.1080/00268976.2019.1650210.

${ }^{31}$ S. Katakura, N. Nishi, K. Kobayashi, K.-i. Amano, and T. Sakka, "Effect of Switching the Length of Alkyl Chains on Electric Double Layer Structure and Differential Capacitance at the Electrode Interface of Quaternary Ammonium-Based Ionic Liquids Studied Using Molecular Dynamics Simulation," The Journal of Physical Chemistry C 124, 7873-7883 (2020), publisher: American Chemical Society.

${ }^{32}$ S. Sharma and H. K. Kashyap, "Structure of Quaternary Ammonium Ionic Liquids at Interfaces: Effects of Cation Tail Modification with Isoelectronic Groups," The Journal of Physical Chemistry C 119, 23955-23967 (2015), publisher: American Chemical Society.

${ }^{33}$ M. M. Perera, M.-W. Lin, H.-J. Chuang, B. P. Chamlagain, C. Wang, X. Tan, M. M.-C. Cheng, D. Tománek, and Z. Zhou, "Improved Carrier Mobility in Few-Layer MoS2 Field-Effect Transistors with Ionic-Liquid Gating," ACS Nano 7, 4449-4458 (2013).

${ }^{34}$ E. Ponomarev, I. Gutiérrez-Lezama, N. Ubrig, and A. F. Morpurgo, "Ambipolar Light-Emitting Transistors on Chemical Vapor Deposited Monolayer MoS2," Nano Letters 15, 8289-8294 (2015), publisher: American Chemical Society.
${ }^{35}$ F. Wang, P. Stepanov, M. Gray, C. N. Lau, M. E. Itkis, and R. C. Haddon, "Ionic Liquid Gating of Suspended MoS2 Field Effect Transistor Devices," Nano Letters 15, 5284-5288 (2015).

${ }^{36}$ H. Tang, W. Niu, F. Liao, H. Zhang, H. Xu, J. Deng, J. Chen, Z. Qiu, J. Wan, Y. Pu, and W. Bao, "Realizing Wafer-Scale and Low-Voltage Operation MoS2 Transistors via Electrolyte Gating," Advanced Electronic Materials 6, 1900838 (2020), eprint: https://onlinelibrary.wiley.com/doi/pdf/10.1002/aelm.201900838.

${ }^{37}$ Y. Zhang, J. Ye, Y. Matsuhashi, and Y. Iwasa, "Ambipolar MoS2 thin flake transistors," 12, 1136-1140, publisher: American Chemical Society.

${ }^{38}$ Y. Xia, W. Xie, P. P. Ruden, and C. D. Frisbie, "Carrier Localization on Surfaces of Organic Semiconductors Gated with Electrolytes," Physical Review Letters 105, 036802 (2010), publisher: American Physical Society.

${ }^{39}$ M. Li, T. Graf, T. D. Schladt, X. Jiang, and S. S. P. Parkin, "Role of Percolation in the Conductance of ElectrolyteGated $\$\{\backslash$ mathrm $\{$ SrTiO $\}\}$ _3 $\$ \$, "$ Physical Review Letters 109, 196803 (2012), publisher: American Physical Society.

${ }^{40}$ W. Zhao, S. Bi, N. Balke, P. D. Rack, T. Z. Ward, S. V. Kalinin, S. Dai, and G. Feng, "Understanding Electric Double-Layer Gating Based on Ionic Liquids: from Nanoscale to Macroscale," ACS Applied Materials \& Interfaces 10, 43211-43218 (2018), publisher: American Chemical Society.

${ }^{41}$ W. Zhao, S. Bi, C. Zhang, P. D. Rack, and G. Feng, "Adding Solvent into Ionic Liquid-Gated Transistor: The Anatomy of Enhanced Gating Performance," ACS Applied Materials \& Interfaces 11, 13822-13830 (2019), publisher: American Chemical Society.

${ }^{42}$ J. B. Haskins and J. W. Lawson, "Evaluation of molecular dynamics simulation methods for ionic liquid electric double layers," The Journal of Chemical Physics 144, 184707 (2016), publisher: American Institute of Physics.

${ }^{43}$ S. Park and J. G. McDaniel, "Interference of electrical double layers: Confinement effects on structure, dynamics, and screening of ionic liquids," The Journal of Chemical Physics 152, 074709 (2020), publisher: American Institute of Physics.

${ }^{44}$ W. Jiang, D. J. Hardy, J. C. Phillips, A. D. MacKerell, K. Schulten, and B. Roux, "High-Performance Scalable Molecular Dynamics Simulations of a Polarizable Force Field Based on Classical Drude Oscillators in NAMD," The Journal of Physical Chemistry Letters 2, 87-92 (2011).

${ }^{45}$ J. Huang, J. A. Lemkul, P. K. Eastman, and A. D. MacKerell Jr., "Molecular dynamics simulations using the drude polarizable force field on GPUs with OpenMM: Implementation, validation, and benchmarks," Journal of Computational Chemistry 39, 1682-1689 (2018).

${ }^{46}$ K. Goloviznina, J. N. Canongia Lopes, M. Costa Gomes, and A. A. H. Pádua, "A Transferable, Polarizable Force Field for Ionic Liquids," Journal of Chemical Theory and Computation (2019), 10.1021/acs.jctc.9b00689.

${ }^{47}$ Z. Wang, Y. Yang, D. L. Olmsted, M. Asta, and B. B. Laird, "Evaluation of the constant potential method in simulating electric double-layer capacitors," The Journal of Chemical Physics 141, 184102 (2014), publisher: American Institute of Physics.

${ }^{48}$ C. Merlet, C. Péan, B. Rotenberg, P. A. Madden, P. Simon, and M. Salanne, "Simulating Supercapacitors: Can We Model Electrodes As Constant Charge Surfaces?" The Journal of Physical Chemistry Letters 4, 264-268 (2013), publisher: American Chemical Society.

${ }^{49}$ K. A. Dwelle and A. P. Willard, "Constant Potential, Electrochemically Active Boundary Conditions for Electrochemical Simulation," The Journal of Physical Chemistry C 123, 24095-24103 (2019).

${ }^{50}$ A. A. H. Pádua, "Resolving dispersion and induction components for polarisable molecular simulations of ionic liquids," The Journal of Chemical Physics 146, 204501 (2017).

${ }^{51}$ J. N. A. Canongia Lopes and A. A. H. Pádua, "Nanostructural organization in ionic liquids," 110, 3330-3335, publisher: American Chemical Society. 
${ }^{52}$ J. I. Siepmann and M. Sprik, "Influence of surface topology and electrostatic potential on water/electrode systems," The Journal of Chemical Physics 102, 511-524 (1995), publisher: American Institute of Physics.

${ }^{53}$ M. K. Petersen, R. Kumar, H. S. White, and G. A. Voth, "A Computationally Efficient Treatment of Polarizable Electrochemical Cells Held at a Constant Potential," The Journal of Physical Chemistry C 116, 4903-4912 (2012), publisher: American Chemical Society.

${ }^{54}$ J. Chmiola, G. Yushin, Y. Gogotsi, C. Portet, P. Simon, and P. L. Taberna, "Anomalous increase in carbon capacitance at pore sizes less than 1 nanometer," 313, 1760-1763, publisher: American Association for the Advancement of Science Section: Report.

${ }^{55}$ T. Fujimoto, M. M. Matsushita, and K. Awaga, "Ionic-liquid component dependence of carrier injection and mobility for electric-double-layer organic thin-film transistors," 116, 52405245, publisher: American Chemical Society.

${ }^{56}$ V. Sresht, A. Govind Rajan, E. Bordes, M. S. Strano, A. A. H. Pádua, and D. Blankschtein, "Quantitative Modeling of MoS2-Solvent Interfaces: Predicting Contact Angles and Exfoliation Performance using Molecular Dynamics," The Journal of Physical Chemistry C 121, 9022-9031 (2017).

${ }^{57}$ K. Goloviznina, Z. Gong, M. Costa Gomes, and A. A. H. Padua, "Transferable, polarizable force field for electrolytes, protic ionic liquids and deep eutectic solvents," (2020).

${ }^{58}$ J. N. Canongia Lopes and A. A. H. Pádua, "CL $\{\&\}$ P: A generic and systematic force field for ionic liquids modeling," Theoretical Chemistry Accounts 131, 1129 (2012).

${ }^{59} \mathrm{~B}$. Hess, "Determining the shear viscosity of model liquids from molecular dynamics simulations," The Journal of Chemical Physics 116, 209-217 (2002).

${ }^{60}$ Z. Gong and H. Sun, "Extension of TEAM Force-Field Database to Ionic Liquids," Journal of Chemical \& Engineering Data 64, 3718-3730 (2019), publisher: American Chemical Society.

${ }^{61} \mathrm{Z}$. Gong and H. Sun, "Pressure-viscosity relation of 2,2,4trimethylhexane predicted using all-atom TEAM force field," Fluid Phase Equilibria 497, 64-70 (2019).

${ }^{62}$ L. Zhao, T. Cheng, and H. Sun, "On the accuracy of predicting shear viscosity of molecular liquids using the periodic perturbation method," The Journal of Chemical Physics 129, 144501 (2008), publisher: American Institute of Physics.

${ }^{63} \mathrm{G}$. Lamoureux and B. Roux, "Modeling induced polarization with classical Drude oscillators: Theory and molecular dynamics simulation algorithm," The Journal of Chemical Physics 119, 30253039 (2003)

${ }^{64}$ C. Y. Son, J. McDaniel, Q. Cui, and A. Yethiraj, "Proper Thermal Equilibration of Simulations with Drude Polarizable Models : Temperature Grouped Dual-Nose-Hoover Thermostat," The Journal of Physical Chemistry Letters (2019), 10.1021/acs.jpclett.9b02983.

${ }^{65}$ G. J. Martyna, M. L. Klein, and M. Tuckerman, "Nosé-Hoover chains: The canonical ensemble via continuous dynamics," The Journal of Chemical Physics 97, 2635-2643 (1992), publisher: American Institute of Physics.

${ }^{66}$ C. M. Neves, K. A. Kurnia, J. A. Coutinho, I. M. Marrucho, J. N. C. Lopes, M. G. Freire, and L. P. N. Rebelo, "Systematic study of the thermophysical properties of imidazolium-based ionic liquids with cyano-functionalized anions," The Journal of Physical Chemistry B 117, 10271-10283 (2013).

${ }^{67}$ M. Karpińska, U. Domańska, and M. Wlazło, "Separation of ethylbenzene/styrene systems using ionic liquids in ternary lle," The Journal of Chemical Thermodynamics 103, 423-431 (2016).

${ }^{68}$ F. Xu, H. Gao, H. Dong, Z. Wang, X. Zhang, B. Ren, and S. Zhang, "Solubility of co2 in aqueous mixtures of monoethanolamine and dicyanamide-based ionic liquids," Fluid Phase Equilibria 365, 80 - 87 (2014).

${ }^{69}$ K. Paduszyński, M. Okuniewski, and U. Domańska, "An effect of cation functionalization on thermophysical properties of ionic liquids and solubility of glucose in them - measurements and pc- saft calculations," The Journal of Chemical Thermodynamics 92 , $81-90$ (2016).

${ }^{70}$ T. Darden, D. York, and L. Pedersen, "Particle mesh Ewald: An $\mathrm{N} \cdot \log (\mathrm{N})$ method for Ewald sums in large systems," The Journal of Chemical Physics 98, 10089-10092 (1993).

${ }^{71}$ J.-P. Ryckaert, G. Ciccotti, and H. J. C. Berendsen, "Numerical integration of the cartesian equations of motion of a system with constraints: molecular dynamics of n-alkanes," Journal of Computational Physics 23, 327-341 (1977).

${ }^{72}$ K.-H. Chow and D. M. Ferguson, "Isothermal-isobaric molecular dynamics simulations with Monte Carlo volume sampling," Computer Physics Communications 91, 283-289 (1995).

${ }^{73}$ J. Åqvist, P. Wennerström, M. Nervall, S. Bjelic, and B. O. Brandsdal, "Molecular dynamics simulations of water and biomolecules with a Monte Carlo constant pressure algorithm," Chemical Physics Letters 384, 288-294 (2004).

${ }^{74}$ P. Eastman, M. S. Friedrichs, J. D. Chodera, R. J. Radmer, C. M. Bruns, J. P. Ku, K. A. Beauchamp, T. J. Lane, L.-P. Wang, D. Shukla, T. Tye, M. Houston, T. Stich, C. Klein, M. R. Shirts, and V. S. Pande, "OpenMM 4: A Reusable, Extensible, Hardware Independent Library for High Performance Molecular Simulation," Journal of Chemical Theory and Computation 9, 461-469 (2013).

${ }^{75}$ B. Hess, C. Kutzner, D. van der Spoel, and E. Lindahl, "GROMACS 4: Algorithms for Highly Efficient, Load-Balanced, and Scalable Molecular Simulation," Journal of Chemical Theory and Computation 4, 435-447 (2008). 